ARTICLE

\title{
Comparison between Energy Straggling Strategy and Continuous Slowing Down Approximation in Monte Carlo Simulation of Secondary Electron Emission of Insulating Materials
}

\author{
Maurizio DAPOR ${ }^{1,2, *}$ \\ ${ }^{1}$ Interdisciplinary Laboratory for Computational Science (LISC), FBK-CMM and University of Trento, \\ via Sommarive 18, I-38123 Povo, Trento, Italy \\ ${ }^{2}$ Department of Materials Engineering and Industrial Technologies, University of Trento, \\ via Mesiano 77, I-38123 Trento, Italy
}

\begin{abstract}
Secondary electrons are commonly used for imaging in scanning electron microscopes, with applications ranging from secondary electron doping contrast in p-n junctions, line-width measurement in critical-dimension scanning electron microscopy and dimensional parameters evaluation in the production of masks and wafers in the semiconductor industry, to the study of biological samples. This paper describes the secondary electron emission yield calculated using two different Monte Carlo approaches. In the first, based on the energy straggling strategy, one takes into account all the single energy losses suffered by each electron in the secondary electron cascade. This method has been demonstrated to be very accurate for the calculation of the secondary electron yield and of the secondary electron energy distribution as well. An alternative way to calculate the secondary electron yield is based on a continuous slowing down approximation and uses as input the electron stopping power of the material being considered. As this work demonstrates that the secondary electron yields calculated using the two approaches are very close and in agreement with the experiment, the much faster continuous slowing down approximation is recommended. On the other hand, if other physical quantities, such as the secondary electron distributions, are required, the energy straggling strategy should be preferred, even if it requires much longer CPU times, due to its stronger physical background.
\end{abstract}

KEYWORDS: Monte Carlo, secondary electron emission, energy straggling, continuous slowing down approximation

\section{Introduction}

Secondary electron emission is a process where primary incident electrons impinging on a surface induce the emission of secondary electrons. The number of the emitted secondary electrons divided by the number of the incident electrons is the so-called secondary electron emission yield. The secondary electron emission yield is measured as the integral of the secondary electron energy distribution over the energy range from 0 to $50 \mathrm{eV}$.

The Monte Carlo (MC) calculation of the secondary electron emission yield can be performed either taking into account all the details of the many mechanisms of the electron energy loss, ${ }^{1-3)}$ or assuming a continuous slowing down approximation. $\left.{ }^{4-8}\right)$ The use of the first approach has stronger physical basis but, due to the detailed description of all the collisions in the secondary electron cascade, it corresponds to a scheme very time consuming. The continuous slowing down approximation represents instead an approach which saves a lot of CPU time. Its physical foundation is, on the other hand, more questionable. This paper reports about the MC simulations of the secondary electron emission from polymethylmethacrylate (PMMA), silicon dioxide and alu-

*Corresponding author, E-mail:dapor@fbk.eu

(c) 2011 Atomic Energy Society of Japan, All Rights Reserved. minum oxide, insulating materials with many technological applications, obtained with the two approaches. It demonstrates that, if we limit ourselves to the calculation of the yield as a function of the primary energy, the two Monte Carlo schemes give equivalent results for any practical purposes.

Secondary electrons are used for imaging in scanning electron microscopes, with applications ranging from secondary electron doping contrast in p-n junctions, ${ }^{9-13)}$ line-width measurement in critical-dimension scanning electron microscopy, ${ }^{14-19)}$ to the study of biological samples. ${ }^{20,21)}$

The MC scheme based on the energy straggling strategy takes into account all the single energy losses suffered by each electron in the secondary electron cascade. ${ }^{1-3,9,18)}$ This method is very accurate for the calculation of the secondary electron yield and energy distribution as well. ${ }^{1-3,9,11,12,18,19)}$ The mentioned alternative MC scheme to calculate the secondary electron yield, based on a continuous slowing down approximation, uses as input the electron stopping power of the material being considered. ${ }^{6-8)}$

The secondary electron yields calculated using the two approaches are very close. What is more, the two MC schemes give results in satisfactory agreement with the experiment. This means that, for the calculation of the secondary electron yield, the continuous slowing down ap- 
proximation should be preferred, being much faster (more than ten times) than the more detailed scheme. If, on the other hand, secondary electron energy distributions are required, the continuous slowing down approximation cannot be used and the detailed scheme becomes mandatory, even if it is much more CPU time consuming.

\section{Cross Sections}

In order to describe the processes that occur when an electron beam penetrates in a solid target, we need to calculate the elastic and the inelastic collisions suffered by the electrons traveling in the solid. In each collision event the incident electrons both loses energy and changes its traveling direction.

The nuclear collisions, due to the large mass difference between the electron and the atomic nucleus, are nearly elastic: they strongly affect the direction of the incident electron, while the energy transfer is negligible.

The energy dissipation of the incident electron mainly occurs through atomic electron excitations or ejections, plasmon excitations, phonons creation and annihilation, and electron-polaron interactions. These scattering processes also influence the electron trajectory in the solid.

\section{Elastic Scattering}

The differential elastic-scattering cross section calculation of electrons interacting with free and bound atoms requires numerical quantum-mechanical calculations. The elastic scattering process was treated by calculating the phase shifts. Since the large-radial coordinate asymptotic behavior of the wave function is known, the phase shifts can be computed by solving the Dirac's equation for a central electrostatic field up to a large radius for which the atomic potential can be safely ignored (Mott cross section: Relativistic partial wave expansion method ). For a recent review of the method, see Yablonski et al. ${ }^{22)}$ Details of the present calculations can be found in Dapor. ${ }^{23,24)}$ The atomic potential used for the elastic scattering calculation was that of Hartree-Fock. Since electrons are identical particles, the exchange was included in the calculations, as low-energy elastic scattering was treated: indeed, the incident electron may be captured by an atom with emission of a new electron. When the target atom is bound in a solid, the outer electronic orbitals of the atom are modified. In order to take into account such a change, solid-state effects have to be introduced. To describe solid-state effects, the muffin-tin model was used in which the potential of each atom of the solid is altered by the nearest-neighbor atoms.

\section{Inelastic Scattering and Dielectric Function}

The inelastic interactions with the target electrons is described by the dielectric function. ${ }^{25}$ For a recent review of the subject, see Taioli et al. ${ }^{26)}$ In the Hartree atomic units where $\hbar=m=e=1$ ( $\hbar$ is the Planck's constant divided by $2 \pi, m$ the electron mass, and $e$ the electron charge), the inverse inelastic mean free path $\lambda^{-1}$ inel and the stopping power $-d E / d s$ are given, respectively, by

$$
\begin{aligned}
& \lambda_{\text {inel }}^{-1}=\int p(E, \omega) d \omega, \\
& -\frac{d E}{d s}=\int \omega p(E, \omega) d \omega,
\end{aligned}
$$

with integrations extended over all the allowed values of the energy transfer $\omega$. Here the function $p(E, \omega)$ is the probability for energy loss $\omega$ per unit distance traveled by an electron of energy $E$. If $q$ is the momentum transfer and $\varepsilon(q, \omega)$ is the complex dielectric function describing the response of the medium, then $p(E, \omega)$ is given by

$$
p(E, \omega)=\frac{1}{\pi E} \int_{q_{-}}^{q_{+}} \frac{d q}{q} \operatorname{Im}\left[\frac{-1}{\varepsilon(q, \omega)}\right],
$$

where

$$
q_{ \pm}=2(\sqrt{E} \pm \sqrt{E-\omega}),
$$

Using the above theory, Ashley has shown that the inverse inelastic mean free path and the stopping power can be well approximated, respectively, by

$$
\begin{aligned}
& \lambda_{\text {inel }}^{-1}=\frac{1}{2 \pi E} \int_{0}^{w_{\max }} \operatorname{Im}\left[\frac{-1}{\varepsilon(0, \omega)}\right] L\left(\frac{\omega}{E}\right) d \omega, \\
& -\frac{d E}{d s}=\frac{1}{\pi E} \int_{0}^{w_{\max }} \operatorname{Im}\left[\frac{-1}{\varepsilon(0, \omega)}\right] S\left(\frac{\omega}{E}\right) \omega d \omega,
\end{aligned}
$$

where

$$
\begin{aligned}
& L(x)=(1-x) \ln \frac{4}{x}-\frac{7}{4} x+x^{3 / 2}-\frac{33}{32} x^{2}, \\
& S(x)=\ln \left(\frac{1.166}{x}\right)-\frac{3}{4} x-\frac{x}{4} \ln \left(\frac{4}{x}\right)+ \\
& +\frac{1}{2} x^{3 / 2}-\frac{x^{2}}{16} \ln \left(\frac{4}{x}\right)-\left(\frac{31}{48}\right) x^{2},
\end{aligned}
$$

and $W_{\max }=E / 2$ is the maximum energy transfer. ${ }^{27)}$

\section{Generation of Phonons}

When their energy becomes lower than $20 \mathrm{eV}$, another energy loss and angular deflection mechanism for the electrons traveling in an insulating material is represented by the electron-phonon interaction. For the present work we have calculated it according to the Fröhlich theory. ${ }^{28)}$ Fröhlich considered, in particular, the interaction of free conduction electrons with the longitudinal optical mode lattice vibrations. The interaction with the lattice corresponds to the absorption and to the generation of phonons. As the phonon generation probability, corresponding to an electron energy loss $\mathrm{W}_{\mathrm{ph}}$, is much higher than the phonon absorption probability, the last can be safely neglected.

\section{Generation of Polarons}

The generation of a polaron by a low-energy electron moving in an insulating material corresponds to an induced polarization field that has a stabilizing effect on the moving electron: the polaron is a quasi-particle with a relevant effec- 
tive mass moving in a dielectric medium, consisting of an electron, or a hole created in the valence band, with its polarization cloud around it. According to Ganachaud and Mokrani the inverse inelastic mean free path for a low-energy electron to be trapped in the ionic lattice is given by

$$
\lambda_{\text {polaron }}^{-1}(E)=C e^{-\gamma E}
$$

where $\mathrm{C}$ and $\gamma$ are constants depending on the material. ${ }^{1)}$

\section{Monte Carlo Schemes}

\section{Energy Straggling Strategy}

If $r$ is a random number uniformly distributed in the interval $[0,1]$, every step length $\Delta s$ of each electron traveling in the solid is calculated assuming the Poisson statistics, so that $\Delta s=-\lambda \ln r$. In this equation, $\lambda$ is the electron mean free path including all the scattering mechanisms involved. Its reciprocal, i.e. the so called inverse inelastic mean free path, can be expressed as the sum of all the inverse mean free paths of the interactions of the electrons with the target: in particular it is necessary to take into account the inverse mean free path relative to the elastic interactions among the incident electrons and the screened atomic nuclei, $\lambda^{-1}$ el, that relative to the inelastic interactions among the incident electrons and the atomic ones, $\lambda^{-1}$ inel, that relative to the electron-phonon interactions, $\lambda^{-1}$ ph, and that relative to the electron-polaron interaction, $\lambda^{-1}$ polaron, so that $\lambda^{-1}=\lambda^{-1}$ el + $\lambda^{-1}$ inel $+\lambda^{-1}$ phonon $+\lambda^{-1}$ polaron. Details about this Monte Carlo strategy can be found in Ganachaud and Mokrani, ${ }^{1)}$ and in Dapor et al. ${ }^{18)}$ Using random numbers one establishes the kind of collision. If the collision is inelastic, the energy loss is calculated according to the specific inelastic scattering cross section (electron-electron, electron-phonon, or electron-polaron). If the collision is elastic, the scattering angle is calculated according to the Mott cross section.

When a secondary electron reaches the target surface, it can be emitted only if its energy $E$ and its direction $\vartheta$ with respect to the normal to the surface satisfy the condition

$$
E \cos \vartheta \geq \xi
$$

where $\xi$ is electron affinity, i.e. the potential energy barrier between the vacuum level and the minimum of the conduction band. The transmission coefficient $T$ is given by

$$
T=\frac{4 \sqrt{1-\xi /\left(E \cos ^{2} \vartheta\right)}}{\left[1+\sqrt{1-\xi /\left(E \cos ^{2} \vartheta\right)}\right]^{2}} .
$$

Once calculated the transmission coefficient, which is given by Eq. (11) if the condition (10) is satisfied and zero otherwise, the code generates a random number, $t$, uniformly distributed in the range $(0,1)$. It allows the secondary electron to be emitted into the vacuum if the condition $t<T$ is satisfied. Otherwise, the secondary electron is specularly reflected without energy loss. Notice that the last part of the trajectory of the electrons which are not able to emerge (the specularly reflected ones) is followed by the code as well, as they can reach the surface again with the energy and angle necessary to emerge. Furthermore, during the last part of their travel, they can contribute to the entire cascade producing ulterior secondary electrons. This Monte Carlo scheme takes into account the entire cascade of secondary electrons.

\section{Continuous Slowing Down Approximation}

The step-length $\Delta s$ is given by $\Delta s=-\lambda_{\mathrm{el}} \ln r$ where $\mathrm{r}$ is a random number uniformly distributed in the range $[0,1]$ and $\lambda_{\mathrm{el}}$ is the elastic mean free path. The energy loss $\Delta E$ along the segment of trajectory $\Delta s$ is approximated by the equation

$$
\Delta E=\frac{d E}{d s} \Delta s,
$$

The electron-atom elastic scattering deflections are calculated using the Mott cross section.

The secondary electron yield is calculated, according to Dionne, ${ }^{4)}$ Lin and Joy, ${ }^{5)}$ Yasuda et al., ${ }^{6)}$ and Walker et al., assuming that (i) the number $d n$ of secondary electrons generated along each step length $d s$, corresponding to the energy loss $d E$, is given by

$$
d n=\frac{1}{\varepsilon_{s}} \frac{d E}{d s} d s=\frac{d E}{\varepsilon_{s}},
$$

where $\varepsilon_{s}$ is the effective energy necessary to generate a single secondary electron and (ii) the probability $P(z)$ that a secondary electron generated at depth $z$ will reach the surface and will emerge from it follows the exponential decay law

$$
P(z)=e^{-z / \lambda_{s}},
$$

where $\lambda_{s}$ is the effective escape depth. Thus the secondary electron emission yield is given by

$$
\delta=\int P(z) d n=\frac{1}{\varepsilon_{s}} \int e^{-z / \lambda_{s}} d E .
$$

\section{Results and Discussion}

The MC schemes described above account for the main interactions occurring to the secondary electrons along their travel in insulating targets. ${ }^{1)}$ Statistical errors of both the kind of Monte Carlo calculations are similar and always smaller than $1 \%$.

Even if the physical meaning of the parameters appearing in the empirical laws describing these interactions is clear -so that they are, at least in principle, measureable -- practically they can be determined, at the moment, only through an analysis of their influence on the simulated results and a comparison to the available experimental data.

Using such a kind of analysis, the values of the parameters for PMMA were determined and, in Fig. 1, we have reported the comparison with the available experimental data 29,30) of the simulated results obtained using the detailed Monte Carlo scheme based on the energy straggling strategy (ES scheme in the following). We found out the best fit us- 


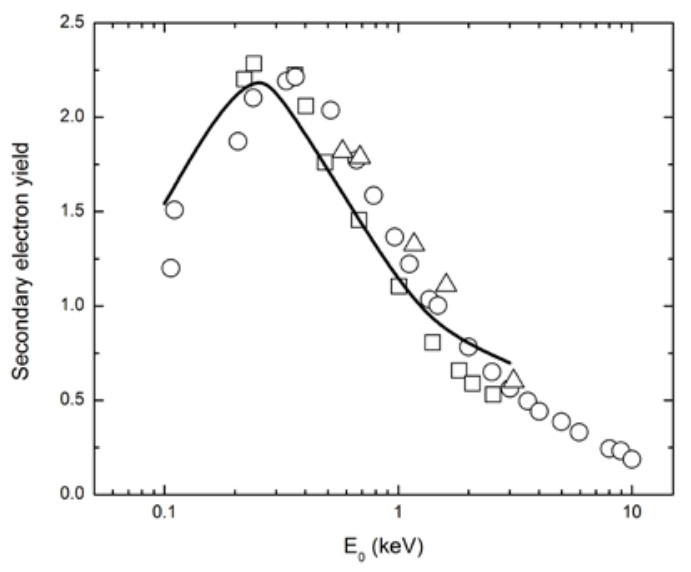

Fig. 1 Comparison between Monte Carlo calculations and experimental data of polymethylmethacrylate (PMMA) secondary electron yield as a function of the primary electron energy. Solid line represents Monte Carlo calculations based on the energy straggling strategy and obtained with $\xi=1.0 \mathrm{eV}, W_{\mathrm{ph}}=0.1 \mathrm{eV}$, $C=1.5 \mathrm{~nm}^{-1}$, and $\gamma=0.14 \mathrm{eV}^{-1}$. Boxes are the Matskevich et al. experimental data, taken from Yasuda et al. ${ }^{6)}$ Circles are the Boubaya and Blaise experimental data. ${ }^{29)}$ Triangles are the Rau et al. experimental data. ${ }^{30)}$

ing a least square fitting procedure with the following values of the parameters: $\xi=1.0 \mathrm{eV}, W_{\mathrm{ph}}=0.1 \mathrm{eV}, C=1.5 \mathrm{~nm}^{-1}$, and $\gamma=0.14 \mathrm{eV}^{-1}$. Notice that, performing a similar analysis, Ganachaud and Mokrani found out, for amorphous $\mathrm{Al}_{2} \mathrm{O}_{3}$, the following values of the not a priori known parameters: $\xi=0.5 \mathrm{eV}, W_{\mathrm{ph}}=0.1 \mathrm{eV}, C=1.0 \mathrm{~nm}^{-1}$, and $\gamma=0.25 \mathrm{eV}^{-1.1)}$ Also note that the yield strongly depends on all these parameters. While both the electron affinity $\xi$ and the electron energy loss due to phonon creation $W_{\text {ph }}$ are quantities that for many materials have been measured and whose values can be found in the scientific literature, less information are available concerning the two parameters characterizing $\mathrm{C}$ and $\gamma$ (relative to the electron-polaron interaction). The Monte Carlo scheme based on the continuous slowing down approximation (CSDA scheme, in the following) also depends on two not a priori known parameters: the effective escape depth, $\lambda_{\mathrm{s}}$, and the effective energy necessary to generate a single secondary electron, $\varepsilon_{\mathrm{s}}$. Using the ES curve determined by the comparison with the experimental data presented in Fig. 1, it is possible to determine the values of $\lambda_{\mathrm{s}}$ and $\varepsilon_{\mathrm{s}}$ of PMMA which correspond to the least square best fit.

The procedure is described in Figs. 2 and 3.

In Fig. 2, in particular, the value of $\lambda_{\mathrm{s}}$ was set to $1.0 \mathrm{~nm}$. The comparison between the CSDA and ES results is shown, with $\varepsilon_{\mathrm{s}}$ ranging from $6 \mathrm{eV}$ to $9 \mathrm{eV}$.

As the best value of $\varepsilon_{\mathrm{s}}$ is $7.5 \mathrm{eV}$, Fig. 3 reports the comparison corresponding to $\varepsilon_{\mathrm{s}}=7.5 \mathrm{eV}$ and allowing $\lambda_{\mathrm{s}}$ to range between 0.5 and $1.5 \mathrm{~nm}$.

The comparisons of the results of the CSDA code to the available experimental data ${ }^{29-33)}$ for $\mathrm{PMMA} \mathrm{SiO}_{2}$, and $\mathrm{Al}_{2} \mathrm{O}_{3}$ are shown in Figs. 4, 5, and 6, respectively.

The values of the parameters, reasonably in agreement

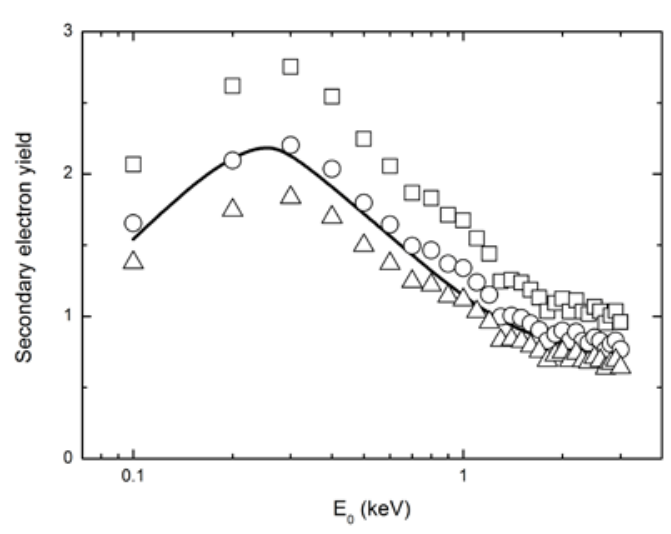

Fig. 2 Comparison between Monte Carlo calculations of PMMA secondary electron yield as a function of the primary electron energy. Solid line represents Monte Carlo calculations based on the energy straggling strategy (see Fig. 1 for details). Symbols are Monte Carlo calculations based on the continuous slowing down approximation and obtained with $\lambda_{\mathrm{s}}=1.0 \mathrm{~nm}$ and $\varepsilon_{\mathrm{s}}=6.0 \mathrm{eV}$ (squares), $\varepsilon_{\mathrm{s}}=7.5 \mathrm{eV}$ (circles), $\varepsilon_{\mathrm{s}}=9.0 \mathrm{eV}$ (triangles).

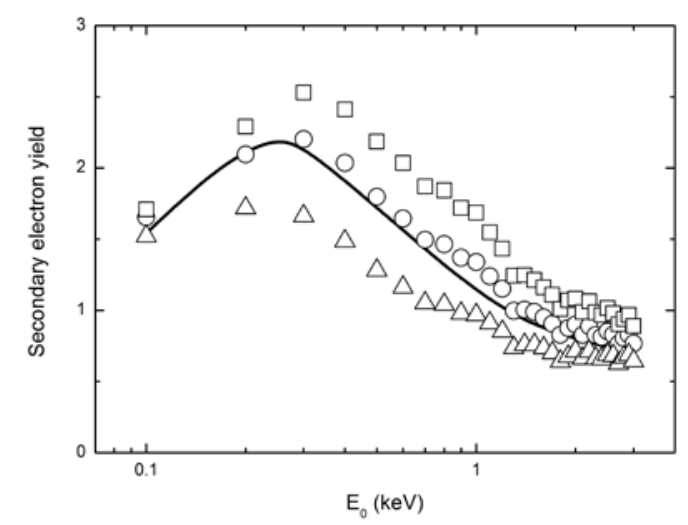

Fig. 3 Comparison between Monte Carlo calculations of PMMA secondary electron yield as a function of the primary electron energy. Solid line represents Monte Carlo calculations based on the energy straggling strategy (see Fig. 1 for details). Symbols are Monte Carlo calculations based on the continuous slowing down approximation and obtained with $\varepsilon_{\mathrm{s}}=7.5 \mathrm{eV}$ and $\lambda_{\mathrm{s}}=1.5 \mathrm{~nm}$ (squares), $\lambda_{\mathrm{s}}=1.0 \mathrm{~nm}$ (circles), $\lambda_{\mathrm{s}}=0.5 \mathrm{~nm}$ (triangles).

with other physics reference data ${ }^{1,5)}$, were determined in order to get the least square best fit of the results of the CSDA code to the experimental data. They are collected in Table $\mathbf{1 .}$ In Tables 2, 3, and 4, the calculated $\chi^{2}$ values, considered to quantitatively evaluate the agreement between the CSDA Monte Carlo simulated data (obtained using the parameters in Table 1) and the examined experimental data are reported, along with the number $v$ of degrees of freedom utilized for each comparison. For the reader convenience, we have also reported the lower critical values of the $\chi^{2}$ distribution for any given $v$, along with the corresponding probability ( $p=0.99)$ of exceeding these critical values. As all the calculated $\chi^{2}$ are significantly smaller than the critical ones, this means that, in the hypothesis that the Monte Carlo data 
Table 1 Values of the effective escape depth, $\lambda_{s}$, and of the effective energy necessary to generate a single secondary electron, $\varepsilon_{s}$, obtained in order to get the best fit of the Monte Carlo code, based on the continuous slowing down approximation, to the available experimental data concerning the secondary electron emission yield.

\begin{tabular}{ccc}
\hline Material & $\begin{array}{c}\lambda_{\mathrm{s}} \\
(\mathrm{nm})\end{array}$ & $\begin{array}{c}\varepsilon_{\mathrm{s}} \\
(\mathrm{eV})\end{array}$ \\
\hline PMMA & 1.0 & 7.5 \\
\hline $\mathrm{SiO}_{2}$ & 0.5 & 12.0 \\
\hline $\mathrm{Al}_{2} \mathrm{O}_{3}$ & 1.5 & 6.0 \\
\hline
\end{tabular}

Table 2 PMMA: comparison between the calculated $\chi^{2}$ and the lower critical values of $\chi^{2}$ distribution corresponding to a probability of $99 \%$. As the calculated $\chi^{2}$ are significantly smaller than the lower critical value of $\chi^{2}$ distribution, the discrepancies between CSDA Monte Carlo data and experimental results can be attributed with high probability (greater than 99\%) to statistical fluctuations, so that we can conclude that the CSDA Monte Carlo data corresponding to the parameters in Table 1 very well approximate the considered experimental data.

\begin{tabular}{ccccc}
\hline & $\begin{array}{c}\text { Calcu- } \\
\text { lated } \chi^{2}\end{array}$ & $\begin{array}{c}\text { Number } v \\
\text { of degrees } \\
\text { of freedom }\end{array}$ & $\begin{array}{c}\text { Probability } \\
p \text { of } \\
\text { exceeding } \\
\text { the critical } \\
\text { value }\end{array}$ & $\begin{array}{c}\text { Lower } \\
\text { critical } \\
\text { value of } \chi^{2} \\
\text { distribution }\end{array}$ \\
\hline $\begin{array}{c}\text { Matskevich } \\
\text { et al. }{ }^{6}\end{array}$ & 0.476 & 11 & 0.99 & 3.053 \\
\hline $\begin{array}{c}\text { Boubaya } \\
\text { and Blaise }^{29)}\end{array}$ & 0.466 & 16 & 0.99 & 5.812 \\
\hline Rau et al. $^{30)}$ & 0.111 & 4 & 0.99 & 0.297 \\
\hline
\end{tabular}

approximate the experimental ones (the so-called null hypothesis), there is a probability greater than the $99 \%$ that the observed discrepancies are due to statistical fluctuations. Similar results were found out also comparing the experimental results to the ES Monte Carlo simulated data.

The time of computation necessary to the ES code is much higher than the time of computation necessary to the CSDA code. For a typical simulation (1 keV electrons impinging on PMMA), the CSDA scheme is more than ten times faster than the ES one. For example, with a personal computer (2.83 GHz processor) for the case of the evaluation of the secondary electron yield of PMMA, using $10^{5}$ primary electrons and a primary electron energy of $1 \mathrm{keV}$, we found out that ES strategy requires more than 300 seconds of computation, while the CSDA code only spends 25 seconds of CPU time. The reason of this great difference in CPU time is related to the secondary electron cascade. The ES MC strategy requires that the entire cascade is followed. The CSDA MC code, on the other hand, is able to establish the number of secondary electrons produced at each step of every primary electron trajectory. Notice that a further advantage of the CSDA MC strategy is the reduced number of not a priori known physical parameters (only two against the four quantities required by the energy straggling strategy).

Of course the ES MC code is based on a stronger physical
Table $3 \quad \mathrm{SiO}_{2}$ : comparison between the calculated $\chi^{2}$ and the lower critical values of $\chi^{2}$ distribution corresponding to a probability of $99 \%$. As the calculated $\chi^{2}$ are significantly smaller than the lower critical value of $\chi^{2}$ distribution, the discrepancies between CSDA Monte Carlo data and experimental results can be attributed with high probability (greater than 99\%) to statistical fluctuations, so that we can conclude that the CSDA Monte Carlo data corresponding to the parameters in Table 1 very well approximate the considered experimental data.

\begin{tabular}{ccccc}
\hline & $\begin{array}{c}\text { Calcu- } \\
\text { lated } \chi^{2}\end{array}$ & $\begin{array}{c}\text { Number } v \\
\text { of degrees } \\
\text { of freedom }\end{array}$ & $\begin{array}{c}\text { Probability } \\
p \text { of } \\
\text { exceeding } \\
\text { the critical } \\
\text { value }\end{array}$ & $\begin{array}{c}\text { Lower } \\
\text { critical } \\
\text { value of } \chi^{2} \\
\text { distribution }\end{array}$ \\
\hline Dionne $^{31)}$ & 0,0366 & 6 & 0.99 & 0,872 \\
\hline $\begin{array}{c}\text { Joy and } \\
\text { Joy }\end{array}$ & 0,164 & 11 & 0.99 & 3,053 \\
\hline
\end{tabular}

Table $4 \quad \mathrm{Al}_{2} \mathrm{O}_{3}$ : comparison between the calculated $\chi^{2}$ and the lower critical values of $\chi^{2}$ distribution corresponding to a probability of $99 \%$. As the calculated $\chi^{2}$ are significantly smaller than the lower critical value of $\chi^{2}$ distribution, the discrepancies between CSDA Monte Carlo data and experimental results can be attributed with high probability (greater than 99\%) to statistical fluctuations, so that we can conclude that the CSDA Monte Carlo data corresponding to the parameters in Table 1 very well approximate the considered experimental data.

\begin{tabular}{lcccc}
\hline & $\begin{array}{c}\text { Calcu- } \\
\text { lated } \chi^{2}\end{array}$ & $\begin{array}{c}\text { Number } v \\
\text { of degrees } \\
\text { of freedom }\end{array}$ & $\begin{array}{c}\text { Probability } \\
p \text { of } \\
\text { exceeding } \\
\text { the critical } \\
\text { value }\end{array}$ & $\begin{array}{c}\text { Lower } \\
\text { critical } \\
\text { value of } \chi^{2} \\
\text { distribution }\end{array}$ \\
\hline Dawson $^{33)}$ & 0,905 & 11 & 0.99 & 3,053 \\
\hline
\end{tabular}

background and allows one to calculate other important properties such as the secondary electron energy distribution, ${ }^{2,3,9,11,12)}$ and the lateral, angular, and depth distributions $^{18)}$ which are not accessible using the CSDA approximation.

The advantage in using the CSDA code, in practical terms, with respect to just performing an empirical fit to the experimental data is related, of course, to other predictive capabilities of the Monte Carlo simulations. If it is, indeed, certainly true that, at the moment, the CSDA model requires a fit to existing data or to the results of the detailed simulation to calculate its free parameters, one should take into account that if the parameters were known for a large number of materials, they could be used for investigating many problems, different from the one we have used to find out the values of the parameters; such as, for example, the dependence of the secondary electron yield on the angle of incidence for any given primary energy, or the secondary electron emission from unsupported thin films (on both sides of the film), or the secondary electron emission yield from thin films deposited on bulk of different materials, and so on. Of course, all these possibilities, which will be the subject of further investigations, are not accessible to a simple empirical fit to the experimental data. 


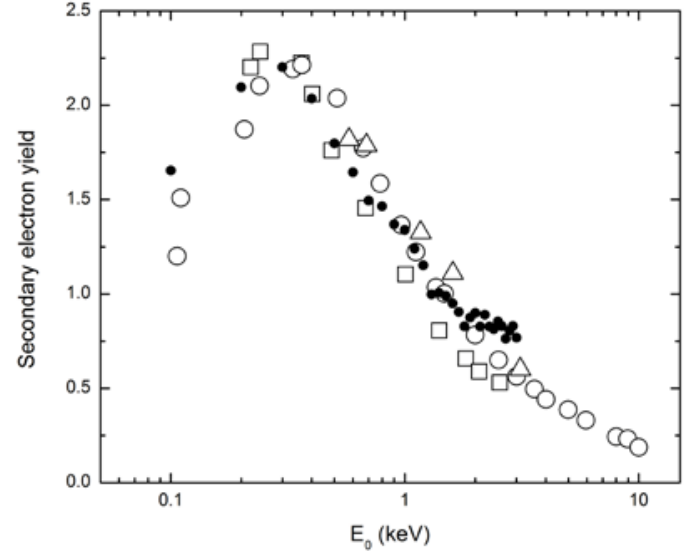

Fig. 4 Comparison between Monte Carlo calculations and experimental data of PMMA secondary electron yield as a function of the primary electron energy. Filled circles represent Monte Carlo calculations based on the continuous slowing down approximation and obtained with $\lambda_{\mathrm{s}}=1.0 \mathrm{~nm}$ and $\varepsilon_{\mathrm{s}}=7.5 \mathrm{eV}$. Empty boxes are the Matskevich et al. experimental data, taken from Yasuda et al. ${ }^{6)}$ Empty circles are the Boubaya and Blaise experimental data. ${ }^{29)}$ Empty triangles are the Rau et al. experimental data. ${ }^{30)}$

In conclusion, the very fast CSDA MC code is recommended for the calculation of the secondary electron yield. If the secondary electron distributions are required, the ES MC strategy should be preferred, even if it requires much longer CPU time.

\section{Conclusion}

An analysis of the results of two different Monte Carlo approaches (energy straggling scheme and continuous slowing down approximation scheme) for the determination of the yield of the secondary electrons emitted by insulating targets, in particular PMMA, $\mathrm{SiO}_{2}$ and $\mathrm{Al}_{2} \mathrm{O}_{3}$, was presented. The two approaches give similar results for what concerns the secondary electron emission yield as a function of the electron primary energy. Furthermore the simulated results are in excellent agreement with the available experimental data. The CSDA code is much faster than the ES code, so that it is recommended, also for its simplicity of implementation and for the reduced number of a priori not known physical parameters (just two against the four necessary to the ES code). If, on the other hand, secondary electron distributions are required, the ES strategy should be preferred, even if it requires much longer CPU time.

\section{Acknowledgment}

This paper incorporates the results of many discussions with friends and colleagues. The author is grateful, in particular, to Giovanni Garberoglio (LISC-FBK, Trento), Stefano Gialanella (University of Trento), and Simone Taioli (LISC-FBK, Trento) for their very useful, helpful, and stimulating suggestions.

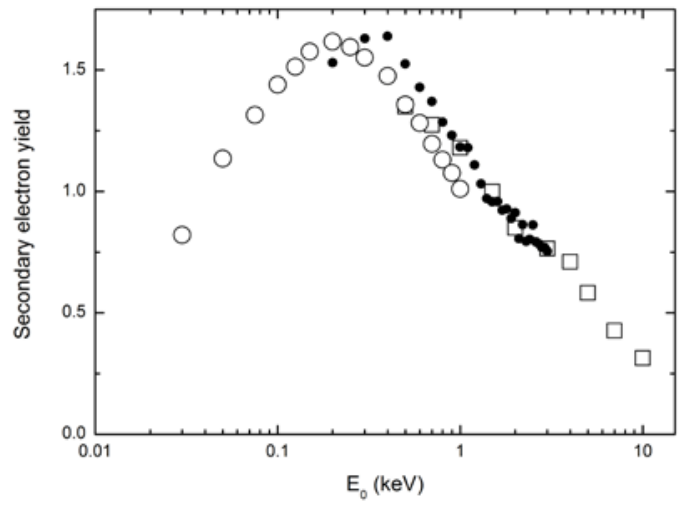

Fig. 5 Comparison between Monte Carlo calculations and experimental data of $\mathrm{SiO}_{2}$ secondary electron yield as a function of the primary electron energy. Filled circles represent Monte Carlo calculations based on the continuous slowing down approximation and obtained with $\lambda_{\mathrm{s}}=0.5 \mathrm{~nm}$ and $\varepsilon_{\mathrm{s}}=12.0 \mathrm{eV}$. Empty circles are the Dionne experimental data. ${ }^{31)}$ Empty boxes are the Joy and Joy experimental data, taken from the Joy's database. $^{32)}$

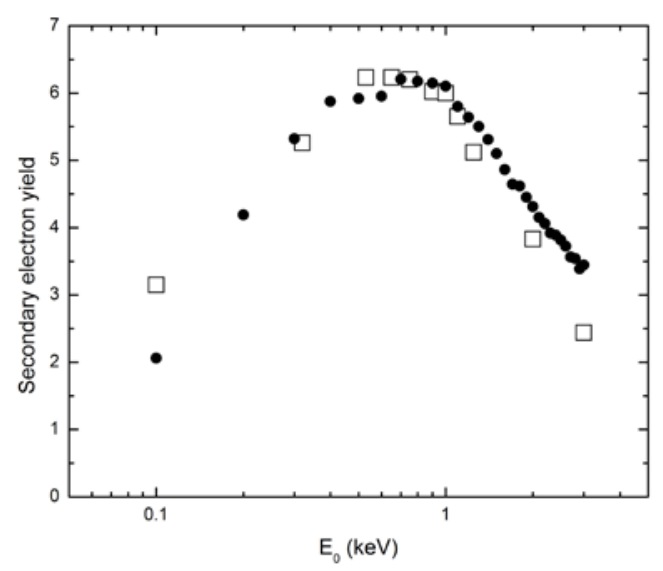

Fig. 6 Comparison between Monte Carlo calculations and experimental data of $\mathrm{Al}_{2} \mathrm{O}_{3}$ secondary electron yield as a function of the primary electron energy. Filled circles represent Monte Carlo calculations based on the continuous slowing down approximation and obtained with $\lambda_{\mathrm{s}}=1.5 \mathrm{~nm}$ and $\varepsilon_{\mathrm{s}}=6.0 \mathrm{eV}$. Empty boxes are the Dawson experimental data. ${ }^{33)}$

\section{References}

1) J. P. Ganachaud, A. Mokrani, "Theoretical study of the secondary electrom emission of insulating targets," Surf. Science, 334, 329-341 (1995).

2) J. Ch. Kuhr, H. J. Fitting, "Monte Carlo simulation of electron emission from solids," J. Electron Spectrosc. Relat. Phenom., 105, 257-273 (1999).

3) M. Dapor, “A Monte Carlo investigation of secondary electron emission from solid targets: Spherical symmetry versus momentum conservation within the classical binary collision model,” Nucl. Instr. Meth. Phys. Res., B267, 3055-3058 (2009).

4) G. F. Dionne, "Effects of secondary electron scattering on secondary emission yield curves," J. Appl. Phys., 44, 5361-5364 (1973). 
5) Y. Lin, D. C. Joy, "A new examination of secondary electron yield data,” Surf. Interface Anal., 37, 895-900 (2005).

6) M. Yasuda, K. Morimoto, Y. Kainuma, H. Kawata, Y. Hirai, "Analysis of charging phenomena of polymer films on silicon substrates under electron beam irradiation,” Jpn. J. Appl. Phys., 47, 4890-4892 (2008).

7) C. G. H. Walker, M. M. El-Gomati, A. M. D. Assa'd, M. Zadražil, "The secondary electron emission yield for 24 solid elements excited by primary electrons in the range 250-5000 eV: A theory/experiment comparison," Scanning, 30, 365-380 (2008).

8) M. Dapor, A. Miotello, D. Zari, "Monte Carlo simulation of positron-stimulated secondary electron emission from solids,” Phys. Rev., B61, 5979-5986 (2000).

9) M. Dapor, B. J. Inkson, C. Rodenburg, J. M. Rodenburg, “A comprehensive Monte Carlo calculation of dopant contrast in secondary-electron imaging," Europhys. Lett., 82, 30006 (5 pp) (2008).

10) C. G. H. Walker, F. Zaggout, M. M. El-Gomati, "The role of oxygen in secondary electron contrast in doped semiconductors using low voltage scanning electron microscopy,” J. Appl. Phys., 104, 123713 (6 pp) (2008).

11) M. Dapor, M. A. E. Jepson, B. J. Inkson, C. Rodenburg, “The effect of oxide overlayers on secondary electron dopant mapping," Microsc. Microanal., 15, 237-243 (2009).

12) C. Rodenburg, M. A. E. Jepson, E. G. T. Bosch, M. Dapor, "Energy selective scanning electron microscopy to reduce the effect of contamination layers on scanning electron microscope dopant mapping,” Ultramicroscopy, 110, 1185-1191 (2010).

13) I. Volotsenko et al., "Secondary electron doping contrast: Theory based on scanning electron microscope and Kelvin probe force microscopy measurements,” J. Appl. Phys., 107, 014510 (7 pp) (2010).

14) C. G. Frase, D. Gnieser, H. Bosse, "Model-based SEM for dimensional metrology tasks in semiconductor and mask industry,” J. Phys. D: Appl. Phys., 42, 183001 (17 pp) (2009).

15) J. S. Villarrubia, Z. J. Ding, "Sensitivity of SEM width measurements to model assumptions," Proceedings of SPIE, The International Society for Optical Engineering, 7272, 72720R (15 pp) (2009).

16) H. Abe, S. Babin, S. Borisov, A. Hamaguchi, A. Ivanchikov, M. Kadowaki, Y. Yamazaki, "Time dependence of SEM signal due to charging: measurements and simulation using Monte-Carlo software," Proceedings of SPIE, The International Society for Optical Engineering, 7272, 727248 (9 pp) (2009).

17) M. Kadowaki, A. Hamaguchi, H. Abe, Y. Yamazaki, S. Borisov, A. Ivanchikov, S. Babin, "Investigation of factors causing difference between simulation and real SEM image," Proceedings of SPIE, The International Society for Optical
Engineering, 7272, 72723I (9 pp) (2009).

18) M. Dapor, M. Ciappa, W. Fichtner, "Monte Carlo modeling in the low-energy domain of the secondary electron emission of polymethylmethacrylate for critical-dimension scanning electron microscopy,” J. Micro/Nanolith. MEMS MOEMS, 9, 023001 (9 pp) (2010).

19) A. Koschik, M. Ciappa, S. Holzer, M. Dapor, W. Fichtner, “A novel Monte Carlo simulation code for line-width measurement in critical-dimension scanning electron microscopy," Proceedings of SPIE, The International Society for Optical Engineering, 7729, 77290X (12 pp) (2010).

20) T. Ogura, "A high contrast method of unstained biological samples under a thin carbon film by scanning electron microscopy,” Biochem. Biophys. Res. Comm., 377, 79-84 (2008).

21) T. Ogura, "Analyzing indirect secondary electron contrast of unstained bacteriophage T4 based on SEM images and Monte Carlo simulations," Biochem. Biophys. Res. Comm., 380, 254-259 (2009).

22) A. Jablonski, F. Salvat, C. J. Powell, “Comparison of electron elastic-scattering cross sections calculated from two commonly used atomic potentials,” J. Phys. Chem. Ref. Data, 33, 409-451 (2004).

23) M. Dapor, "Elastic scattering calculations for electrons and positrons in solid targets," J. Appl. Phys., 79, 8406-8411 (1996).

24) M. Dapor, Electron-Beam Interactions with Solids: Application of the Monte Carlo Method to Electron Scattering Problems, Springer, Berlin, 2003.

25) R. H. Ritchie, "Interaction of charged particles with a degenerate Fermi-Dirac electron gas”, Phys. Rev., 114, 644-654 (1959).

26) S. Taioli, S. Simonucci, L. Calliari, M. Dapor, "Electron spectroscopies and inelastic processes in nanoclusters and solids: theory and experiment,” Phys. Rep., 493, 237-319 (2010).

27) J. C. Ashley, "Interaction of low-energy electrons with condensed matter: stopping powers and inelastic mean free paths from optical data,” J. Electron Spectrosc. Relat. Phenom., 46, 199-214 (1988).

28) H. Fröhlich, "Electrons in lattice fields," Adv. Phys., 3, 325-364 (1954).

29) M. Boubaya, G. Blaise, "Charging regime of PMMA studied by secondary electron emission,” Eur. Phys. J.: Appl. Phys., 37, 79-86 (2007).

30) É. I. Rau, E. N. Evstaf'eva, M. V. Adrianov, “Mechanisms of charging of insulators under irradiation with medium-energy electron beams,” Phys. Solid State, 50, 599-607 (2008).

31) G. F. Dionne "Origin of secondary-electron-emission yield-curve parameters,” J. Appl. Phys., 46, 3347-3351 (1975).

32) D. C. Joy, "Electron solid interaction database," http://web.utk.edu/ srcutk/htm/interact.htm (2008).

33) P. H. Dawson, "Secondary electron emission yields of some ceramics,” J. Appl. Phys., 37, 3644-3665 (1966). 